\title{
la instrucción
} especial

\section{para estructuras de hormigón armado}

\author{
ALVARO GARCIA MESEGUER \\ Ingeniero de Caminos
}

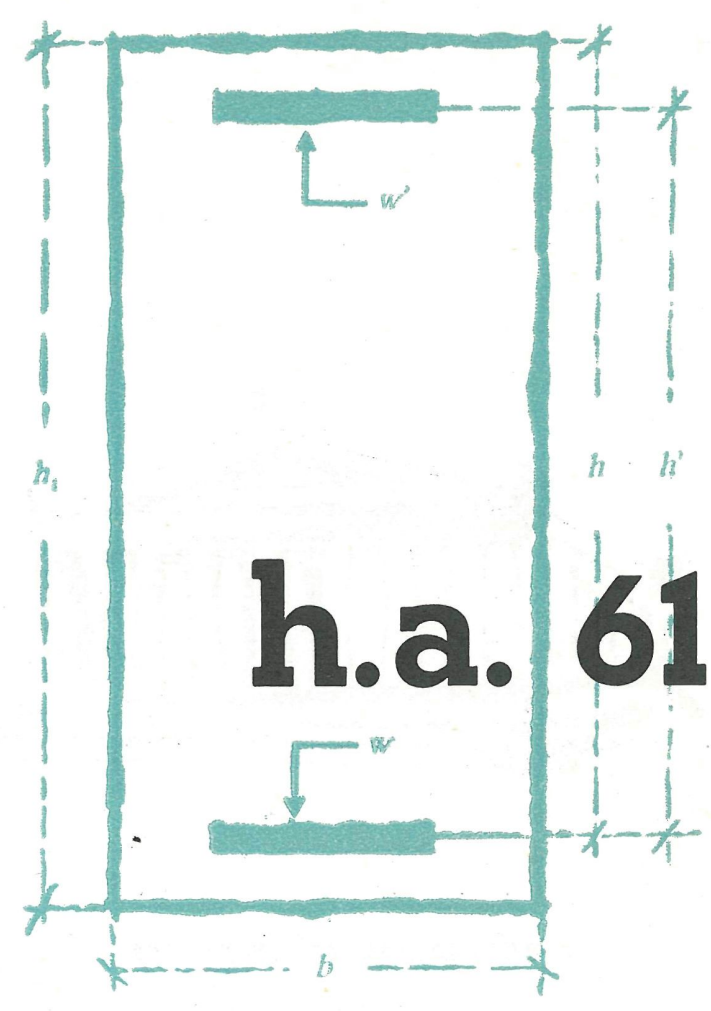

El Instituto ha publicado, recientemente, las partes segunda y tercera de la Instrucción especial para estructuras de hormigón armado, «H.A.61», que se refieren a Documentos del Proyecto $\mathrm{y}$ a Cálculo de estructuras lineales planas, respectivamente. Dicha Instrucción, comparada con las que habitualmente se vienen utilizando, entraña tantas novedades que no es aventurado imaginar una reaceión inicialmente hostil por parte de técnicos y proyectistas; cosa bien lógica, a nuestro modo de ver, puesto que, desde pocas veces encuentra el asidero de un término o de una idea que coincida con lo que él tiene ya muy trabajado y sabido.

El objeto del presente artículo es adentrar en la H.A.61-o mejor, divagar por ella-, llevados de la mano de un ejemplo muy sencillo. Tendremos, así, ocasión de comentar algunos puntos, a la vez que comparamos los resultados con los obtenidos utilizando el método clásico.

Se trata de dimensionar una viga rectangular, simplemente apoyada, con los siguientes datos de partida:

Resistencia media del hormigón: $R_{\mathrm{m}}=180 \mathrm{~kg} / \mathrm{cm}^{2}$.

Sección de la viga: $h_{\mathrm{t}}=50 \mathrm{~cm} ; b=18 \mathrm{~cm}$.

Luz: $\mathrm{L}=9 \mathrm{~m}$.

Sobrecarga: $q_{1}=0,75 \mathrm{t} / \mathrm{m}$. 


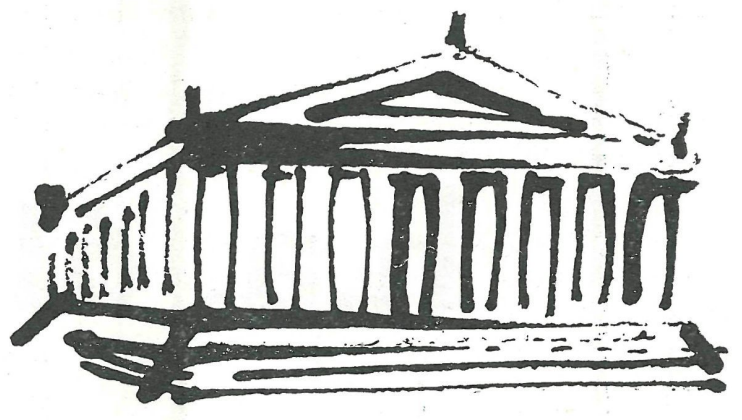

método clásico

(Instrucción O. P. 44)

Peso propio:

$$
q_{2}=0,5 \times 0,18 \times 2,5=0,225 \mathrm{t} / \mathrm{m} .
$$

Carga total:

$$
p=q_{1}+q_{2}=0,975 \mathrm{t} / \mathrm{m} .
$$

Coeficiente de seguridad respecto al hormigón:

$$
\mathrm{C}=3 \text {. }
$$

Resistencia de cálculo del hormigón:

$$
R_{\mathrm{c}}=\frac{180}{3}=60 \mathrm{~kg} / \mathrm{cm}^{2}
$$

Momento máximo (en el centro):

$$
\frac{1}{8} \times 0,975 \times 9^{2}=9,87 \mathrm{~m} \cdot \mathrm{t}
$$

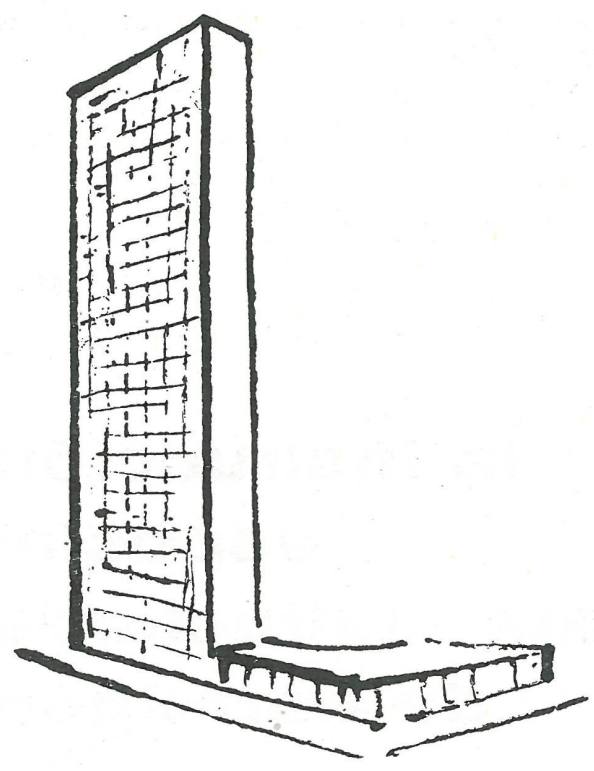

\section{cálculo de rotura}

(Instrucción H. A. 61)

Peso propio:

$$
q_{2}=0,5 \times 0,18 \times 2,5=0,225 \mathrm{t} / \mathrm{m} .
$$

Carga total:

$$
p=q_{1}+q_{2}=0,975 \mathrm{t} / \mathrm{m} .
$$

Coeficiente de mayoración:

$$
\mathrm{C}_{\mathrm{M}}=1,65 \text {. }
$$

Carga mayorada:

$$
\underline{p}=1,65 \times 0,975=1,61 \mathrm{t} / \mathrm{m} .
$$

Resistencia característica del hormigón:

$$
R_{\mathrm{k}}=0,85 \times 180=153 \mathrm{~kg} / \mathrm{cm}^{2} .
$$

Resistencia minorada del hormigón:

$$
\underline{\mathrm{R}}=\frac{153}{1,6}=95,6 \mathrm{~kg} / \mathrm{cm}^{2}
$$

Momento máximo (en el centro) :

$$
\underline{M}=\frac{1}{8} \times 1,61 \times 9^{2}=16,3 \mathrm{~m} \cdot \mathrm{t}
$$


Hacemos el primer alto, porque ya han aparecido conceptos "raros" y palabras "raras", y fuerza es comentarlos.

Lo primero que choca son los términos "mayorar" y "minorar". Acudimos al diccionario de la Real Academia, y encontramos, como deflnición de minorar: "disminuir, acortar o reducir a menos una cosa". Vamos bien. En el cálculo se va.a introducir una resistencia disminuída o reducida, $\$$ ponde a ello.

Con la palabra mayorar tenemos menos suerte, pues el diccionario nos dice que es un vocablo antiguo, que significa: "dar en mayor o mejor porción"; mientras que lo que aquí quiere significarse es: "aumentar, alargar o ampliar a más una cosa". Desgraciadamente, una definición así - precisamente opuesta a la de minorar-no existe en nuestro diccionario: la $H$. A. 61 se ha tomado la libertad de adjudicarla al verbo mayorar. Bien puede perdonarse tal licencia. Máxime cuando con ello se sugiere la idea justa: en el cálculo debe introducirse una carga mayor que la real.

Dejando de lado las cuestiones lingüísticas, vamos con los conceptos. El coeficiente de mayoración elegido, $\mathbf{C}_{\mathrm{M}}=1,65$, corresponde, según el artículo 3.7.1, al caso en que se prevé que las consecuencias del hundimiento pueden ser muy graves (teatros, tribunas, etc.). Nos hemos colocado, pues, en el extremo más desfavorable; lo cual es honrado a la hora de comparar métodos (entre paréntesis: esta variación del coeficiente de mayoración con la magnitud de los daños es mucho más lógica que la imperturbabilidad del coeficiente $\mathbf{C}=3$ del método clásico).

La resistencia característica del hormigón se ha obtenido suponiendo una dispersión $\delta=15 \%$. Entonces, $R_{k}=R_{m}(1-\delta)=0,85 R_{m}$. Esta suposición no habría hecho falta si el dato de partida para este ejemplo hubiese sido, tal como propugna la $\mathrm{H}$. A. 61, la resistencia característica en vez de la resistencia media; es decir, si, a la hora de ensayar las probetas, en vez de calcular la media aritmética de todas ellas $\left(R_{m}\right)$, se hubiese calculado la media aritmética de la mitad más baja de resultados $\left(R_{k}\right)$. Es oportuno, en conclusión, apuntar otro tanto a favor de la H. A. 61, ya que, con su modo de introducir la resistencia del hormigón, tiene en cuenta la dispersión del material; cosa que olvida el método clásico (¿Confiaría usted lo mismo en un hormigón cuyas probetas, ensayadas a compresión, diesen como resultado las cifras 180 y 20, que en otro cuyas probetas diesen 102 y 98?).

Ahora surge una pregunta de los partidarios de la columna izquierda: ¿Por qué esa notación tan incómoda, subrayando letras? Más aún: ¿Qué necesidad había de cambiar de nombre, en la notación, a cosas que toda la vida se han escrito de otro modo, como, por ejemplo, el límite elástico del acero?

Efectivamente, estamos completamente de acuerdo en que el tema notación es importantísimo. Tanto, que hay toda una Comisión en el seno del Comité Europeo del Hormigón, encargada de tan espinoso asunto, y aún no ha llegado a una conclusión definitiva. Por eso, la H. A. 61 se ha planteado este problema en todo su rigor. $Y$ ha partido de la idea de que un buen cuerpo de notación debe reunir las características siguientes, citadas por orden de preferencia:

- ser lógico y consecuente consigo mismo;

- ser lo más sencillo posible, sin prestarse a confusiones;

- admitir el empleo de máquina de escribir;

- conservar al máximo lo establecido por la costumbre.

Si esto se acepta, hay que utilizar un número minimo de letras griegas; hay que prescindir de la $l$, que se confunde, a máquina, con el 1 (entonces, la luz será L, y el límite elástico ya no puede ser L); hay que uniformar subíndices, huyendo del doble subíndice; hay que... La notación empleada es la $\mathrm{H}$. A. 61 puede servir. No es, por supuesto, la ideal, y como todo en este mundo, es perfectible. Pero el que sea nueva no debe ser argumento en su contra: si es lógica y funciona, debe imponerse y hacerse costumbre.

En esta línea, el subrayado de algunas letras indica "que se ha tenido en cuenta el margen de seguridad correspondiente". Al ver un momento flector $\mathbf{M}$ subrayado, $\mathbf{M}$, sabemos que ya está mayorado; y hasta parece que, al escribirlo, cuando después se le subraya, uno da testimonio, con ese gesto, de que no se ha olvidado del coeficiente de seguridad.

$Y$ ahora, huelga de filosofías y adelante. 


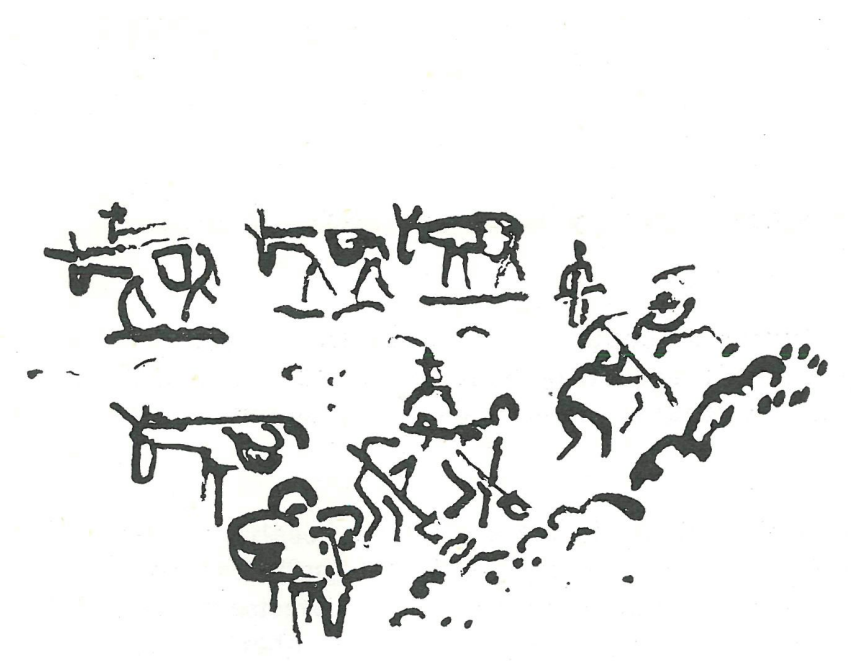

método clásico

(Instrucción O. P. 44)

Se aplican las fórmulas del Löser.

Canto útil crítico:

$$
c=0,095 \sqrt{\frac{9,87}{0,18}}=0,702 \mathrm{~m} .
$$

Como 0,702 $>0,50$, hay que colocar armadura de compresión.

Se supone como canto útil $h=0,455 \mathrm{~m}$.

Momento absorbido:

$$
\mathrm{M}=\frac{0,18 \times 0,455^{2}}{0,009}=4,15 \mathrm{~m} \cdot \mathrm{t}
$$

Momento no absorbido :

$$
\triangle M=9,87-4,15=5,72 \mathrm{~m} . \mathrm{t}
$$

Se supone un recubrimiento de la armadura de compresión $r^{\prime}=4,5 \mathrm{~cm}$.

Armadura de tracción:

$$
s=\frac{0,18 \times 0,455}{0,009}+\frac{5,72}{1,2 \times 0,41}=20,7 \mathrm{~cm}^{2}
$$

equivalentes a $8 \varnothing 18$.

Es válida la suposición hecha para el canto útil.

Armadura de compresión:

$$
a=\frac{5,72}{0,69 \times 0,41}=20,2 \mathrm{~cm}^{2}
$$

equivalentes a $8 \varnothing 18$.

Es válida la suposición hecha para el recubrimiento $r$.

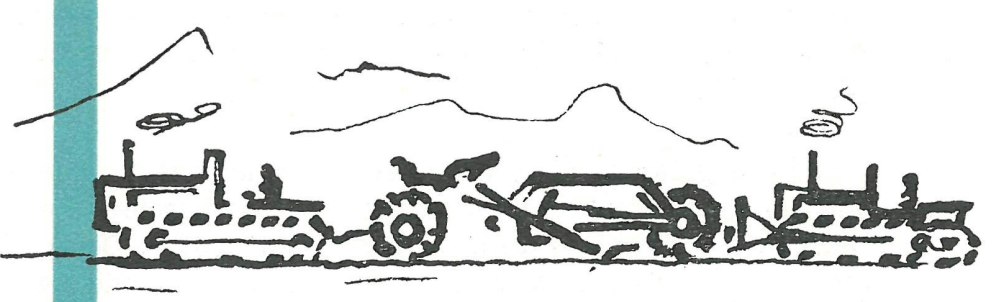

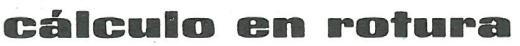

(Instrucción H. A. 61)

Se aplican las fórmulas del Anejo de Cálculo.

Se supone como canto útil $h=0,45 \mathrm{~m}$.

$$
\begin{aligned}
\mathrm{V} & =0,0956 \times 18 \times 45=77,4 \mathrm{t} \\
0,375 \mathrm{~V} h & =0,375 \times 77,4 \times 0,45=13,05 \mathrm{~m} \cdot \mathrm{t}
\end{aligned}
$$

Como $13,05<16,3$, hay que colocar armadura de compresión.

Es de utilización la fórmula [10].

Se supone un recubrimiento de la armadura de compresión $r^{\prime}=3 \mathrm{~cm}$.

Armadura de compresión:

$$
U^{\prime}=\frac{16,3-13,05}{0,42}=7,75 \mathrm{t}
$$

equivalentes a $W^{\prime}=2 \varnothing 16$.

Es válida la suposición hecha para el recubrimiento $r$.

Armadura de tracción:

$$
\mathrm{U}=0,5 \mathrm{~V}+\mathrm{U}^{\prime}=0,5 \times 77,4+7,75=46,45 \mathrm{t}
$$

equivalentes a W=8 $\varnothing 20$.

Es válida la suposición hecha para el canto útil. 
Es momento de detenernos y comentar. En la sección central de la viga se necesitan, según el método clásico, $8 \varnothing 18+8 \varnothing 18=40,8 \mathrm{~cm}^{2}$ de acero; $\mathrm{y}$ según la H. A. $61,8 \varnothing 20+2 \varnothing 16=29,2 \mathrm{~cm}^{2}$ de acero. Es decir, hay un ahorro del $\frac{11,6}{40,8} \times 100=28 \%$.

Salta a la vista la desproporción existente entre ambas armaduras de compresión. La explicación es clara: el cálculo en rotura supone un diagrama rectangular de compresiones en el hormigón, que puede llegar, en profundidad, hasta la mitad del canto útil, como ocurre en este ejemplo. De este modo, el hormigón está aprovechado al máximo. Y así, el momento absorbido por el hormigón es, en la columna derecha, el $80 \%$ del total, mientras que en la columna izquierda es sólo el $42 \%$ del total (*).

Por otra parte, el cálculo clásico supone que la tensión en la armadura de compresión es la que le corresponde por el reparto lineal de tensiones; que en este caso es de $0,69 \times 1.000=690 \mathrm{~kg} / \mathrm{cm}^{2}$. Lo cual significa poco aprovechamiento. Mientras que, en rotura, se supone que esa armadura trabaja a límite elástico (minorado, claro está). Es decir, que está aprovechada al máximo, al igual que la armadura de tracción.

La consecuencia es que, con el nuevo método, se saca ventaja respecto al antiguo, más marcada cuanto más escaso se esté de canto. $Y$ aún en los casos en que, por ser éste holgado, no se requiera armadura de compresión, el cálculo en rotura seguirá proporcionando economía frente al cálculo clásico, por suponer un brazo mecánico mayor, ya que el centro de gravedad del rectángulo (diagrama de tensiones en rotura) está más alto que el del triángulo (diagrama de tensiones clásico), para un mismo volumen de compresiones.

En cuanto a la sencillez de los cálculos, la H. A. 61 se apunta un nuevo tanto sobre su rival. Y seguimos adelante.

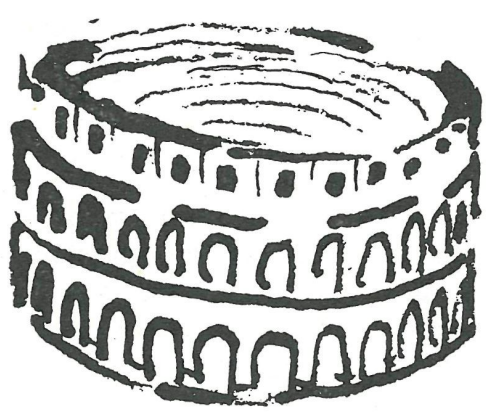

mb̉ำdo clásico

(Instrucción O.P. 44)

Esfuerzo cortante máximo (en apoyos):

$$
\mathrm{Q}=4,5 \times 0,975=4,4 \mathrm{t}
$$

Resistencia del hormigón a esfuerzo cortante:

$$
\mathrm{R}^{\prime}=\frac{1}{2} \sqrt[3]{\mathrm{R}_{m}^{2}}=\frac{1}{2} \sqrt[3]{180^{2}}=16 \mathrm{~kg} / \mathrm{cm}^{2}
$$

Tensión cortante admisible:

$$
\begin{aligned}
\tau_{\operatorname{adm}}=\frac{1}{3} R^{\prime}=5,33 \mathrm{~kg} / \mathrm{cm}^{2} \\
\quad \frac{13,05 \times 100}{16,3}=80 \% ; \frac{4,15 \times 100}{9,87}=42 \%
\end{aligned}
$$

$(*)$

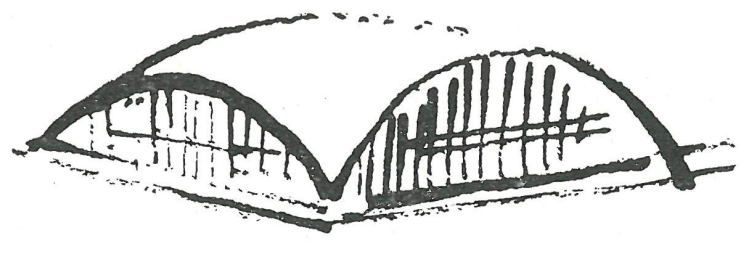

că|cu|co de roture:

(Instrucción H. A. 61)

Esfuerzo cortante máximo (en apoyos):

$$
\underline{\mathrm{T}}=4,5 \times 1,61=7,25 \mathrm{t}
$$

Resistencia virtual del hormigón a esfuerzo cortante:

$$
\underline{R}^{\prime \prime}=0,5 \sqrt{\underline{R}}=0,5 \sqrt{95,6}=4,89 \mathrm{~kg} / \mathrm{cm}^{2}
$$


Tensión cortante:

$$
\tau=\frac{4.400}{\frac{7}{8} \times 47,3 \times 18}=5,9 \mathrm{~kg} / \mathrm{cm}^{2}
$$

Como $\tau>\tau_{\text {adm, }}$ debe disponerse armadura transversal para absorber la totalidad de $\mathbf{Q}$.

Levantando tres parejas de barras a $45^{\circ}$ a partir del apoyo, a distancias entre sí iguales al brazo mecánico, se absorbe:

$$
\mathrm{Q}_{\mathrm{a}}=\frac{1.200 \times 5,1 \times 0,415 \sqrt{2}}{0,415}=8.650 \mathrm{~kg}>\mathrm{Q}
$$

Las barras levantadas dejan de ser eficaces a una distancia del apoyo:

$$
d=2 \times 0,415+\frac{0,415}{2}=1,03 \mathrm{~m}
$$

A esa distancia el esfuerzo cortante vale:

$$
\mathrm{Q}=(4,5-1,03) \times 0,975=3,38 \mathrm{t},
$$

y la tensión cortante,

$$
\tau=\frac{3.380}{\frac{7}{8} \times 47,3 \times 18}=4,54 \mathrm{~kg} / \mathrm{cm}^{2}
$$

menor que la admisible.

No obstante, para evitar el pandeo de la armadura de compresión, deben colocarse cercos $\varnothing 6$ a distancia:

$$
12 \varnothing=12 \times 1,8 \simeq 20 \mathrm{~cm}
$$

Pero como el ancho de la pieza es menor que $20 \mathrm{~cm}$, se colocarán a una distancia igual a:

$$
b=18 \mathrm{~cm}
$$

El hormigón, por sí solo, resiste:

$$
\mathrm{T}_{\mathrm{b}}=\underline{\mathrm{R}} " \mathrm{~b} h=4,89 \frac{18 \times 45}{1.000}=3,96 \mathrm{t}
$$

Como $\mathrm{T}_{\mathrm{b}}<\mathrm{T}$, debe disponerse armadura transversal para absorber la diferencia $\mathbf{T}-\mathbf{T}_{\mathbf{b}}$.

Levantando tres parejas de barras a $45^{\circ}$ a partir del apoyo, a distancias entre sí iguales a vez y media el canto útil, se absorbe (cuadro núm. 5):

$$
\mathrm{T}_{\mathrm{a}}=12 \times 0,85=10,2 \mathrm{t}>\mathrm{T}-\mathrm{T}_{\mathrm{b}}
$$

Las barras levantadas dejan de ser eficaces a una distancia del apoyo:

$$
d=2 \times 0,675+\frac{0,675}{2}=1,68 \mathrm{~m}
$$

A esa distancia el esfuerzo cortante vale:

$$
\underline{T}=(4,5-1,68) \times 1,61=4,54 \mathrm{t} .
$$

La diferencia

$$
\mathrm{T}-\mathrm{T}_{\mathrm{b}}=4,54-3,96=0,58 \mathrm{t}
$$

debe sijsorberse con cercos.

Para no tener que colocar muchos cercos, por efecto de la armadura de compresión, conviene disponer, como tal armadura, $2 \varnothing 18$, en vez de $2 \varnothing 16\left(^{*}\right)$; así, la distancia entre cercos (artículo 3.24.3), puede llegar a valer:

$$
s=10 \varnothing=18 \mathrm{~cm} \text {. }
$$

Con cercos $\varnothing 6$ a esa distancia, se absorbe (cuadro núm. 6):

$$
\mathrm{T}_{\mathrm{a}}=2,92 \mathrm{t}>0,58 \mathrm{t}
$$

Ambos métodos conducen, prácticamente, a la misma solución. Fuera de la zona de barras levantadas, el método clásico no necesita armadura porque la tensión cortante resulta admisible. La H. A. 61 sí las necesita, pero le bastan las mínimas necsarias que hay que disponer por efecto de la armadura de compresión. Véase, pues, cómo hemos procedido con honradez al elegir este ejemplo: si se hubiese elegido otro, con cortantes más fuertes, la H. A. 61 habría sacado ventaja, al utilizar una fórmula aditiva: hormigón y armadura transversal, frente a la fórmula disyuntiva del método clásico: hormigón o armadura transversal.

En resumen: El esquema resultante en cada caso es el siguiente:

- armadura transversal: igual.

- armadura longitudinal: se ahorra un $26 \%$ utilizando H. A. 61 .

- rapidez de cálculo: algo mayor con H. A. 61 .

- Con este cambio, la economía de armadura longitudinal pasa a ser del $26 \%$. 

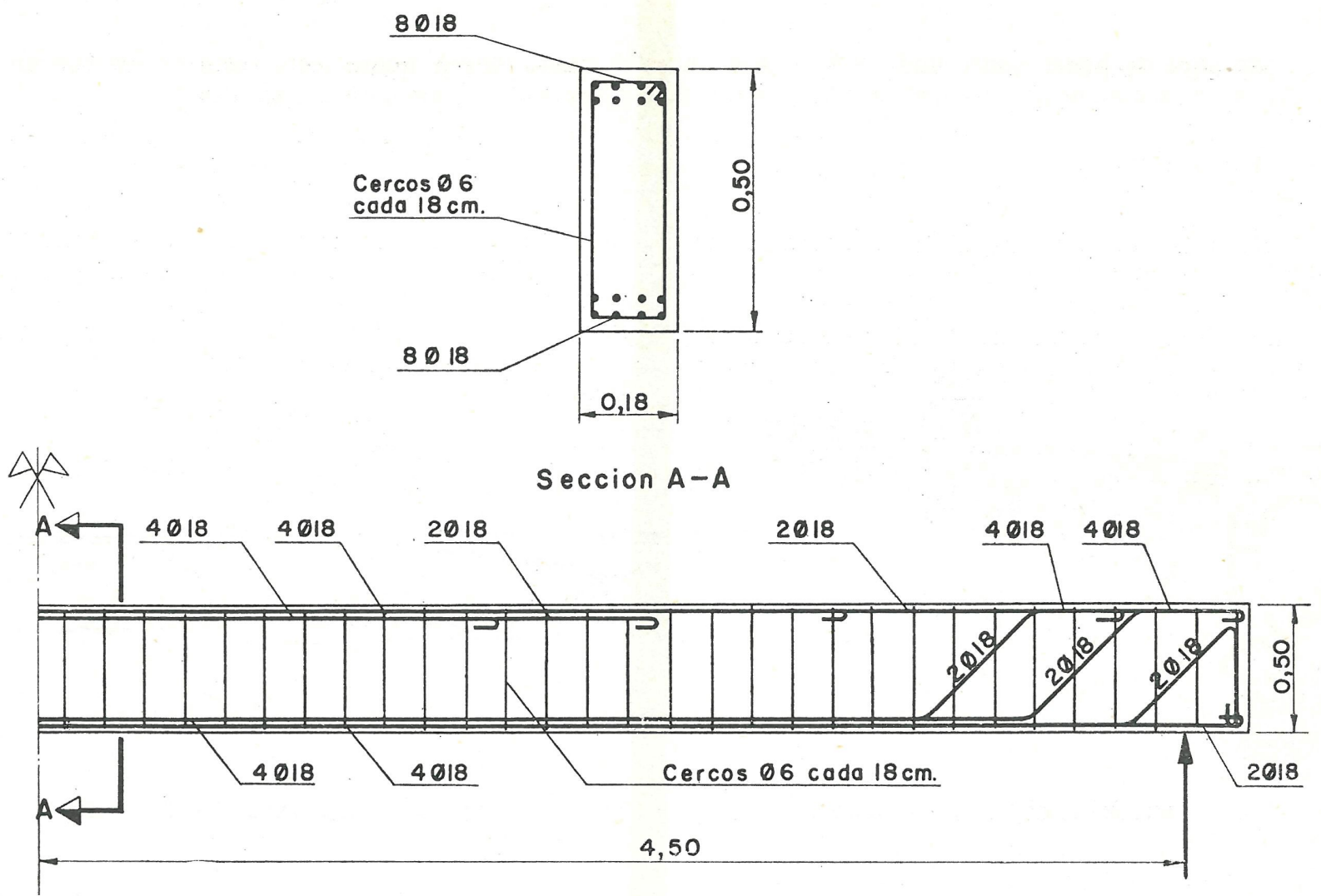

método chlảsico
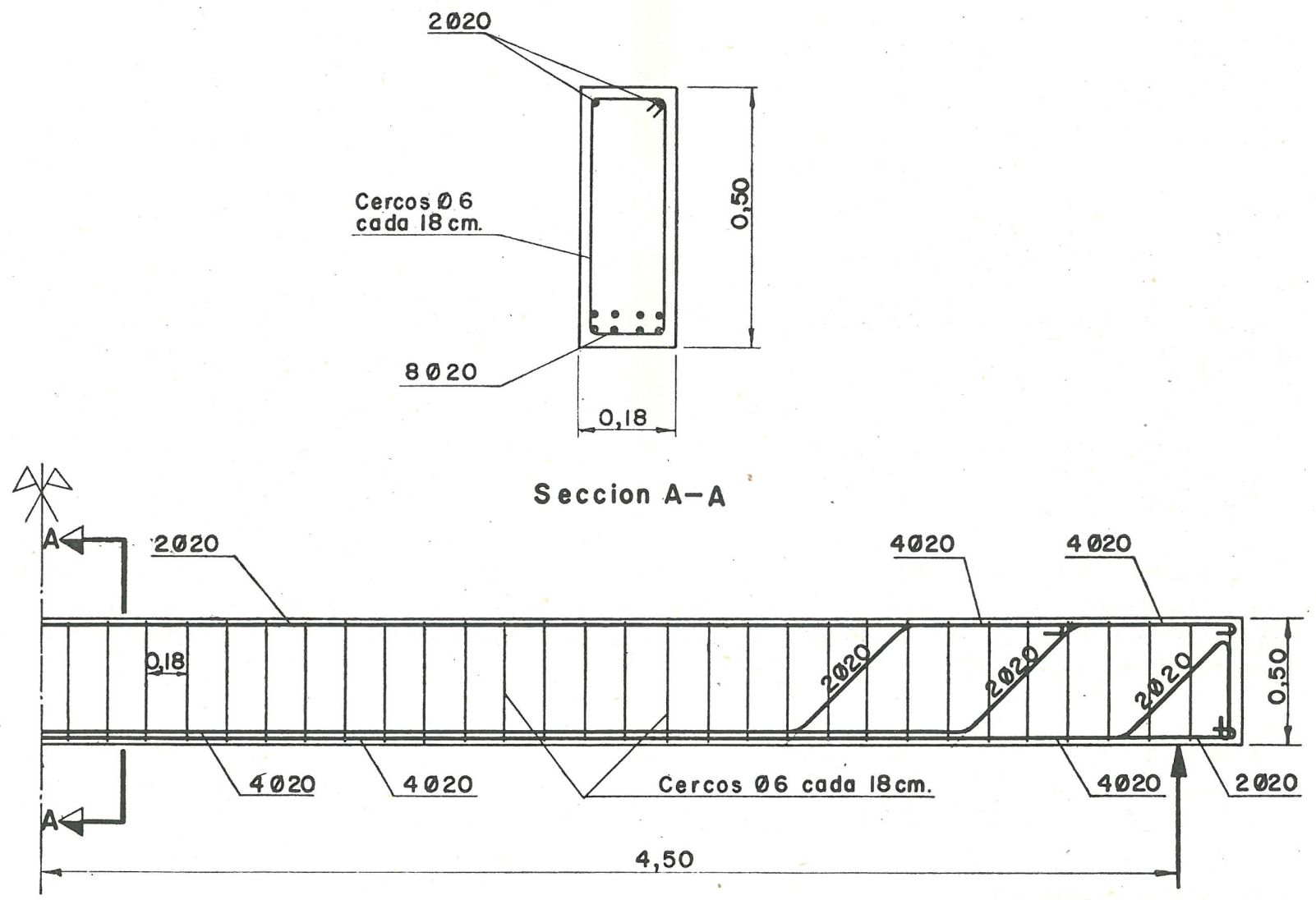

cẳloulo en rotura 
Es.hora de hacer punto final. Pero queda un cabo suelto. Quizá algún lector esté ahora pensando, con sonrisa maliciosa: "iClaro! ¡Se ahorra un $26 \%$ a costa del coeficiente de seguridad!"

Vamos a verio.

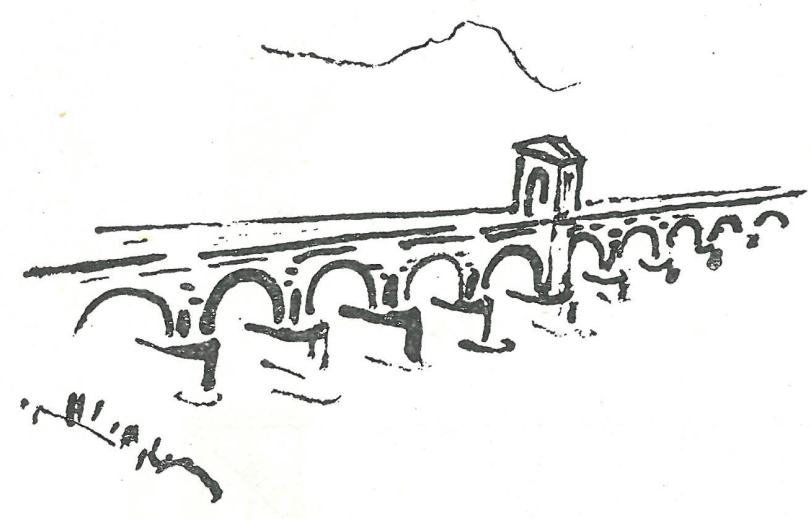

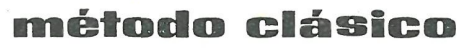

(Instrucción O.P. 44)

Coeficiente de seguridad respecto al hormigón:

$$
\mathrm{C}_{\mathrm{b}}=\mathbf{3}
$$

Coeficiente de seguridad respecto al acero:

$$
\mathrm{C}_{\mathrm{a}}=2
$$

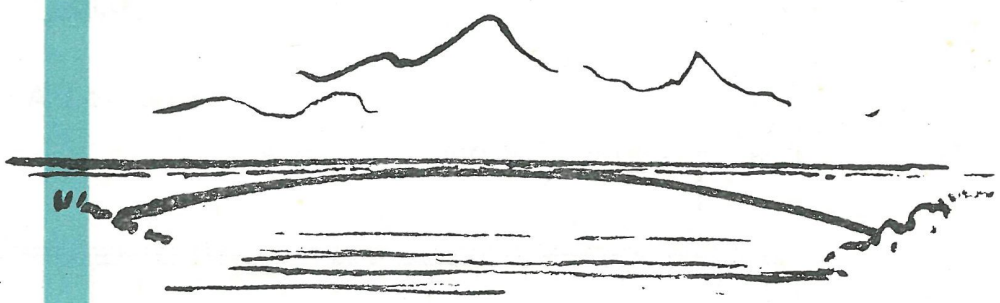

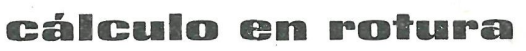

(Instrucción H. A. 61)

Coeficiente de seguridad respecto al hormigón:

$$
\mathrm{C}_{\mathrm{b}}=\frac{1,65 \times 1,6}{0,85}=3,11
$$

Coeficiente de seguridad respecto al acero:

$$
\mathrm{C}_{\mathrm{a}}=1,65 \times 1,2=1,98 .
$$

La seguridad es, prácticamente, la misma. Con la ventaja de que, el nuevo método, desdobla el criterio de seguridad en dos parámetros: el de mayoración, que multiplica las cargas, y el de minoración, que divide las resistencias de los materiales. Las solicitaciones pésimas de cálculo se deducen de la aplicación de las cargas, mayoradas o no, según sea su efecto desfavorable o favorable. Y los estados tensionales resultantes de ellas, no deben ser superiores a los que la estructura podría soportar con unos materiales que tuviesen, a su vez, resistencias minoradas. El cálculo se lleva a cabo en agotamiento resistente, considerando las leyes reales del comportamiento de los materiales en esas condiciones; con lo que se sabe perfectamente a qué distancia se está de la rotura (gracias a los coeficientes de mayoración y minoración).

Si los materiales fuesen perfectamente elásticos hasta su agotamiento resistente, no habría diferencia entre los resultados del método clásico y los del que ahora se preconiza; pero no siendo esto así, los nuevos criterios adoptados proporcionan dos ventajas importantes: una, la más precisa correlación entre la seguridad real y la calculada; otra, la mayor simplificación y claridad de cálculo...

Para quien haya llegado hasta aquí en su lectura sin el menor asomo de interés, este artículo ha terminado. Para quien no esté en ese caso, este artículo continúa en la propia Instrucción H. A. 61: los últimos párrafos escritos están tomados de sus primeras páginas.

Quien no se emperece ante el estudio de lo nuevo; quien no se deje vencer por la rutina de lo sabido; quien, en fin, haya creído y crea en el magisterio de su autor, Eduardo Torroja, siga adelante. 